\title{
Decision Support System for the Long-Term City Metabolism Planning Problem
}

\author{
M.S.Morley*, D.Vitorino**, K.Behzadian*, R.Ugarelli***, Z.Kapelan*, S.T.Coelho****, M.Do Céu Almeida**** \\ * Centre for Water Systems, University of Exeter, North Park Road, Exeter, EX4 4QF (UK) (M.S.Morley@exeter.ac.uk) \\ ** Addition Lda., Rua Borges Carneiro 34 RC, 1200-619, Lisbon (Portugal) \\ *** SINTEF Building and Infrastructure, Forskningsveien 3b, NO-0314 Oslo (Norway) \\ **** Laboratório Nacional de Engenharia Civil, Av. do Brasil 101, 1700-066, Lisbon (Portugal)
}

\begin{abstract}
A Decision Support System (DSS) tool for the assessment of intervention strategies (Alternatives) in an Urban Water System (UWS) with an integral simulation model called "WaterMet" ${ }^{2}$ " is presented. The DSS permits the user to identify one or more optimal Alternatives over a fixed long-term planning horizon using performance metrics mapped to the TRUST sustainability criteria (Alegre et al., 2012). The DSS exposes lists of in-built intervention options and system performance metrics for the user to compose new Alternatives. The quantitative metrics are calculated by the WaterMet ${ }^{2}$ model and further qualitative or user-defined metrics may be specified by the user or by external tools feeding into the DSS. A Multi-Criteria Decision Analysis (MCDA) approach is employed within the DSS to compare the defined Alternatives and to rank them with respect to a pre-specified weighting scheme for different Scenarios. Two rich, interactive Graphical User Interfaces, one desktop and one webbased, are employed to assist with guiding the end user through the stages of defining the problem, evaluating and ranking Alternatives. This mechanism provides a useful tool for decision makers to compare different strategies for the planning of UWS with respect to multiple Scenarios.

The efficacy of the DSS is demonstrated on a northern European case study inspired by a real-life urban water system for a mixture of quantitative and qualitative criteria. The results demonstrate how the DSS, integrated with an UWS modelling approach, can be used to assist planners in meeting their long-term, strategic level sustainability objectives.
\end{abstract}

Keywords: decision support system, water metabolism model, intervention strategy, risk.

\section{INTRODUCTION}

Urban water systems (UWS) face the long-term perspective of constraints and challenges associated with climate change, urbanisation growth, population growth and the limited availability of natural resources. This prospect requires the adaptation of the operation and infrastructure of UWS to meet uncertain future scenarios through the adoption of mitigating technologies in the water industry.

Before these mitigating options can be practically implemented and incorporated into urban water systems, it is suggested that their performance needs to be simulated, analysed and evaluated with other UWS components through an integrated modelling framework comprising a Decision Support System (DSS). Such Decision Support Systems have received attention from many practitioners and researchers in recent years, leading to the development of tools. A number of recently developed DSSs and software tools for this purpose are AQUACYCLE (Mitchell et al., 2001), UrbanCycle (Hardy et al., 2005), UWOT (Makropoulos et al., 2008), UVQ (Mitchell and Diaper, 2010), CWB (Mackay and Last, 2010), DUWSiM (Willuweit and O'Sullivan, 2013) and DMM (Venkatesh et al., 2014). These models 
typically employ a daily mass-balance based approach to simulate water related fluxes between UWS components in the context of urban water cycle (e.g. clean water, stormwater and wastewater). These DSS tools mainly aim to assess the performance of centralised and decentralised water supply or water demand management options for long term planning of urban water supply and water demand (Willuweit and O'Sullivan, 2013). Each of these DSS tools has some strength points in their developments. Some DSS tools have stressed on water demand modelling at household and neighbourhood areas such as UWOT and UrbanCycle while some other focused on modelling water and other fluxes at system levels such as DMM and DUWSiM. Some of them have focused on analysing the effects of urbanisation scenarios and climate changes on the urban water cycle (Willuweit and O'Sullivan, 2013).

Work Package 54 of the TRUST project is concerned with the development of a DSS which implements a tool which is able to quantify the impact of different sets of interventions/technologies on the performance of an UWS, including associated risks and costs by evaluating a wide variety of sustainability performance metrics under different scenarios. The WaterMet ${ }^{2}$ model (Behzadian et al., 2013a), which undertakes the simulation of the integrated modelling of UWS, is employed in the DSS presented to quantify the key "metabolic" flows in the system.

Two parallel, complementary implementations of the DSS in two different software systems and platforms (i.e. desktop and web-based tools), with feature sets that take advantage of those platforms' specific contexts and target slightly diverse user groups.

\section{METHODS}

For a long-term, strategic-level planning of Urban Water Systems at the city/system level, a number of alternative Intervention Strategies are usually proposed to deal with any possible limitations of the future urban water service. Selection of the most appropriate Intervention Strategy should be considered with respect to a number of different metrics and their preferences specified by stakeholders. Thus, decision making framework is required for evaluating the proposed intervention strategies and comparing them together and finally ranking and selecting the most appropriate one with respect to specified metrics and preferences. Additionally, this selection can be subject to various external scenarios which can affect the evaluation of intervention strategies. All this is handled through the developed DSS in this work package. The developed DSS seeks to support in this is achieved through a novel methodology for comparison and selection of alternative solutions, within the framework of long-term transition paths, and amidst multiple decision criteria.

\section{DSS Implementation}

The assessment of intervention strategies in an UWS is encapsulated in a framework expressed through a DSS. The structure of the classes in the DSS engine is split into three principle modules including Environment, Performance and MCDA. The 'Environment' part manages the specifications of the analysis including timing, intervention strategies, PIs, scenarios and customised model input. The 'Performance' part undertakes the responsibility of evaluating the indicators which are split into two categories: (1) quantitative performance and risk indicators calculated by the WaterMet ${ }^{2}$ and Risk Modules, respectively; (2) qualitative indicators of the aforementioned types, defined within the DSS and quantified by external tools outside the immediate scope of the DSS. Finally, the MCDA module applies a user-configured ranking approach to the specified intervention strategies for the purposes of scoring and ranking them for each scenario and user preference combination. 
In order to configure an evaluation of intervention strategies over a planning horizon in the DSS, the following four principal steps are required from the user: (1) an intervention strategy is defined in the 'Environment' part of the DSS based on the list of available intervention options. The intervention strategy comprises a set of individual interventions, including technologies and their operation on different parts of the UWS, each of which is assumed to occur at a specific time over a defined planning horizon. (2) The PIs of interest to the analysis, including those supported by the WaterMet ${ }^{2}$ model and those supported by other tools outside the DSS, are also specified in the 'Environment' part of the DSS. (3) PIs including performance, risk and cost are evaluated in the 'Performance' section of the DSS. The PIs calculated or supported by the WaterMet ${ }^{2}$ directly such as risk-based indicators are automatically populated in the DSS, whilst others evaluated outside the DSS need to be supplied manually by the user. (4) Scoring and ranking of the defined intervention strategies are conducted in the 'Strategy' part of the DSS by employing a user-defined MCDA.

As a part of the built-in simulation model in the DSS, the WaterMet ${ }^{2}$ model is used to calculate all non risk-based performance indicators in an integrated UWS. This is handled through a simplified approach for modelling water supply, stormwater and wastewater systems based on mass-balance equations. The physical metabolism of this integrated UWS is then quantified through some performance indicators (PIs). Details of the principal flows and storages modelled in WaterMet ${ }^{2}$ as well as descriptions of the components and their functionality can be found in Behzadian et al. (2013a).

The desktop DSS tool (Figure 3) is designed to run under a Windows ${ }^{\mathrm{TM}}$ operating system. It is a standalone software tool, however it will use other deliverables in the TRUST project as the input file. More specifically, the WaterMet ${ }^{2}$ model developed in WP33 can be used in DSS as a simulation model to support the assessment of intervention strategies in an UWS for the long-term planning of UWS.

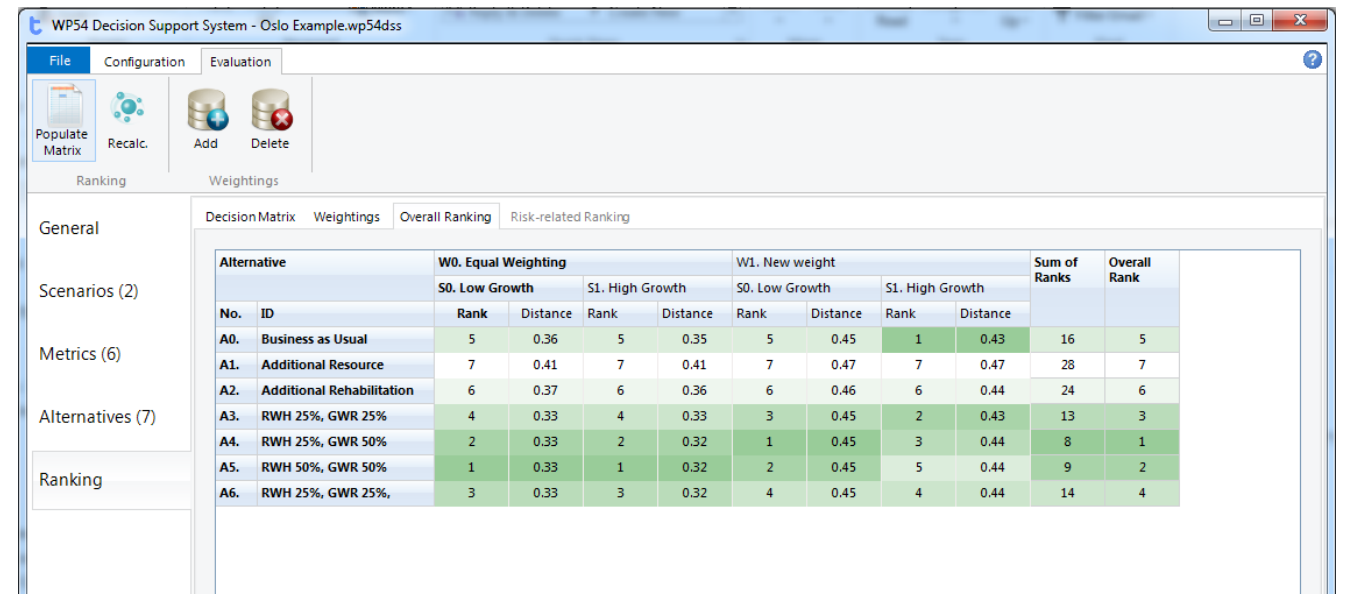

Figure 1 Desktop Tool Interface showing per-Scenario ranking of Alternatives 
The web-based tool (Figure 2) is one of the modules available in Baseform's software deployment for the TRUST Project, alongside the AWARE-P IAM planning software portfolio - a non-intrusive, web-based, collaborative environment targeted at water utility professionals and decision makers. The system has been publicly available since 2012 and has gathered over 1200 registered users worldwide, having been used for IAM plan development in over 50 utilities in Europe, USA and Australia. The web-based tool shares the Baseform platform's visually-oriented interface and usage language, creating a degree of commonality with the available portfolio of tools, namely those developed under TRUST such as the PLAN comparison \& decision tool, aiming specifically at managerial and technical roles in urban water services where decisions impact a number of stakeholders and interests.

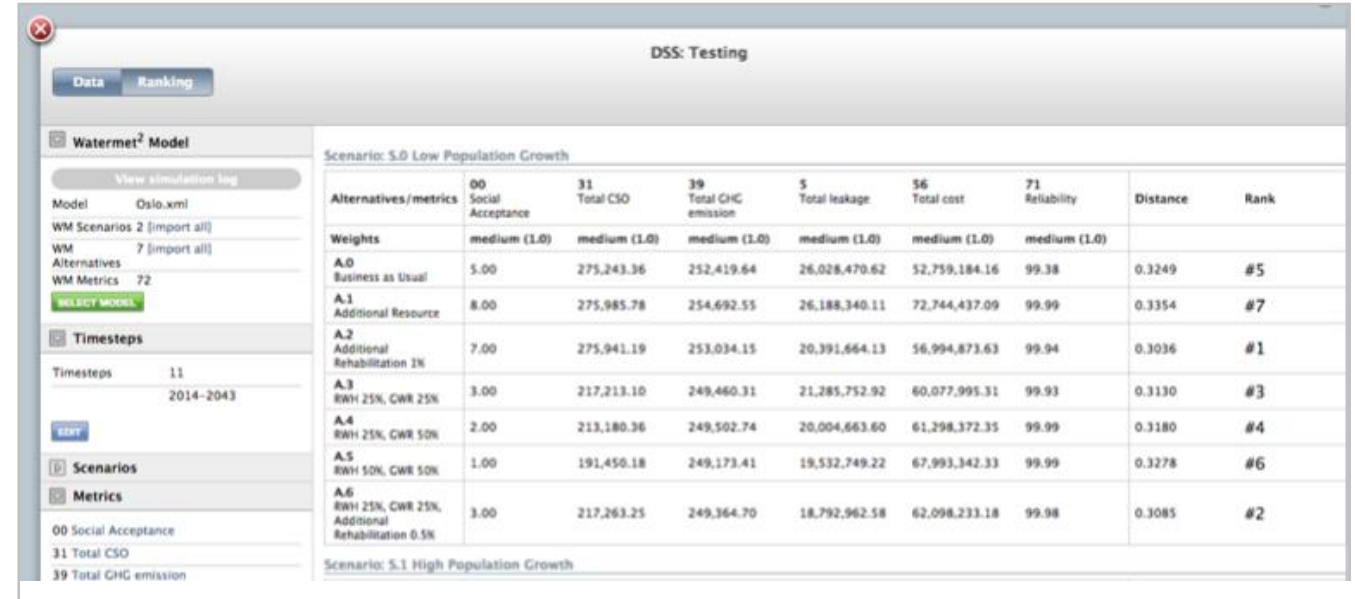

Figure 2 Web-based Interface demonstrating Decision Matrix and per-Scenario ranking of Alternatives

The desktop tool enables additional functionality over and above that available in the web-based tool. In particular, whereas the web-based tool requires that Intervention Strategies (Alternatives) be predefined in the WaterMet ${ }^{2}$ input data, the desktop tool allows the end-user to interactively construct and evaluate their own Alternatives using any combination of the Interventions that are published by the WaterMet ${ }^{2}$ model.

\section{Principal Steps}

\section{Defining the Problem}

Problem definition comprises the specification of three principal components by the user:

1. Analysis Scenarios e.g. different population growth, climate change and other Scenarios which define the external conditions within which the UWS operates;

2. Performance Metrics of the UWS that will be used to assess performance of the system. Metrics may either be those exposed by the WaterMet ${ }^{2}$ metabolism model or user-supplied.

3. Intervention Strategies (or Alternatives). An alternative comprises a set of individual interventions drawn from a predefined list of intervention options supported by the WaterMet ${ }^{2}$ model. Each individual intervention is considered to occur at a specific time within the planning horizon and can have impacts on one or more constituent components of the UWS.

\section{Population of Decision Matrix}

For populating the DSS decision matrix, input data need to be specified and populated first through the relevant DSS forms. By populating scenarios, performance metrics and intervention strategies in the relevant forms, the 'Environment' part of the DSS is completed and becomes ready for the 
'Performance' part of the DSS. Each intervention strategy containing a set of individual intervention options occurring over the planning horizon, each with pre-specified timing needs to be evaluated over the planning horizon. This is effected by modifying the relevant WaterMet ${ }^{2}$ input variables and parameters following the implementation of some intervention(s) and then rerunning the simulation from that point onwards, until the end of planning horizon is reached. Simulation of the UWS is carried out in the DSS using the built-in WatMet ${ }^{2}$ model.

As a result of running the DSS, the quantitative metrics are populated in the DSS decision matrix. The qualitative metrics need to be manually entered in the DSS decision matrix manually. Finally, risk is also calculated in the DSS. Then, setting up the DSS parameters is implemented before running the DSS.

\section{Ranking Alternatives}

Different intervention strategies built by using WaterMet ${ }^{2}$ model or other models need to be compared and ranked with respect to a number of different criteria. Two well-known MCDA methods are implemented in the DSS for the purpose of ranking intervention strategies under different scenarios and user preferences: (a) the Compromise Programming (CP) method (Zeleny, 1973) and the Analytical Hierarchy Process (AHP) method (Saaty, 1980). The two methods were selected because of their widespread use but also because they use different ranking technologies and, also, allow users to express their preferences in a different way. In the CP method, user preferences are specified as multiple evaluation criteria weights making this method more suitable for use by less experienced users. In the AHP method, user preferences are specified via the pairwise criteria-importance comparisons. This requires more experience to configure and employ the method. The DSS will enable the user to select the method to use when solving a particular problem, including the possibility to use both methods on the same problem and then compare results (e.g. to see if there an alternative solution that is ranked highly regardless of the MCDA method used).

\section{RESULTS AND DISCUSSION}

\section{Problem Description}

The case study shown here is inspired by, although not completely representative of, the UWS of a northern European city. This UWS is used here as a reference city for the case study combined with assumptions when necessary (Behzadian \& Kapelan, 2013). The UWS will face a number challenges among which population growth is likely to impose significant strains on the UWS performance for future planning. As a result, it is predicted that the city population with $\sim 750,000$ inhabitants in 2014 is estimated to reach approximately 1,240,000 inhabitants in 2045 based on the highest foreseen rate of population growth.

The DSS tool for the strategic planning of an integrated urban water system (UWS) over a pre-defined long-term planning horizon is presented here. The DSS evaluates and ranks a number of user defined alternative intervention strategies (IS or simply alternatives) by evaluating their impact on a number of (user defined) UWS performance metrics, all for a number of (user defined) scenarios.

\section{Scenarios}

The UWS is likely to face the challenge of population growth in the future which imposes increased water demand on the UWS. Two possible rates of future population growth (i.e. low and high) are postulated for the 30 year planning period starting from 2010. 


\section{Metrics}

According to the performance criteria of sustainability dimensions of water systems (Alegre et al., 2012), six performance metrics are considered for this analysis. These metrics include five quantitative criteria (M0-M4), and a single qualitative criterion, M5. A brief description of these metrics is outlined below:

M0 Reliability of water supply: the ratio of water delivered to customers to the total water demand.

M1 Total cost: annual average of the discounted initial capital investment of interventions plus discounted value of the fixed and variable costs in different UWS components to the first year with a specific discount rate.

M2 GHG emissions: annual average of the aggregated greenhouse gas emissions (GHG), as Global Warming Potential (GWP100) measured in units of carbon dioxide equivalents $\left(\mathrm{CO}_{2}\right.$-eq) from all components of the UWS.

M3 Leakage: Annual average of leakage volume is measured in all conveyance components of water supply assuming leakage is a fixed percentage of water supply in water supply conveyance components.

M4 CSO volume: Annual average of spill volume of CSOs (combined sewer overflow) is measured when daily flow in sewer network exceeds the capacity of a CSO structure.

M5 Social acceptance: the extent to which an intervention strategy would be supported by society, especially water consumers; in order to fulfil the water demands with respect to a number of factors especially safety and health issues.

The main features of these six performance metrics which will be required for the DSS are summarised in Table 1.

Table 1 Specifications of the performance metrics for the numerical example

\begin{tabular}{|c|c|c|c|c|c|c|c|}
\hline \multirow{2}{*}{ ID } & \multirow{2}{*}{ Description } & \multicolumn{3}{|c|}{ Performance Metric } & \multirow{2}{*}{ Quantification } & \multirow{2}{*}{ Goal } & \multirow{2}{*}{$\begin{array}{c}\text { Normalisation } \\
\text { values }\end{array}$} \\
\hline & & Component & Component ID & Units & & & \\
\hline Mo & $\begin{array}{c}\text { Reliability of water } \\
\text { supply/demand } \\
\text { balance }\end{array}$ & UWS & 0 & $\%$ & WaterMet $^{2}$ & $\begin{array}{l}\text { Optimization } \\
\qquad(\max )\end{array}$ & - \\
\hline M1 & Total cost & UWS & 0 & Euros/year & WaterMet $^{2}$ & $\begin{array}{l}\text { Optimization } \\
\text { (min) }\end{array}$ & - \\
\hline M2 & GHG emissions & UWS & 0 & Tons/year & WaterMet $^{2}$ & $\begin{array}{l}\text { Optimization } \\
\text { (min) }\end{array}$ & - \\
\hline M3 & Leakage & UWS & 0 & $\mathrm{~m}^{3} /$ year & WaterMet $^{2}$ & $\begin{array}{l}\text { Optimization } \\
\text { (min) }\end{array}$ & - \\
\hline M4 & CSO volume & UWS & 0 & Tons/year & WaterMet $^{2}$ & $\begin{array}{l}\text { Optimization } \\
\text { (min) }\end{array}$ & - \\
\hline M5 & Social acceptance & UWS & 0 & - & User-specified & $\begin{array}{l}\text { Optimization } \\
\qquad(\max )\end{array}$ & $\begin{array}{c}\operatorname{Min}=1 \\
\operatorname{Max}=10\end{array}$ \\
\hline
\end{tabular}

\section{Alternatives}

To address the above issues, three types of intervention options are proposed for this strategic planning as follows:

1. Addition of a new water resource along with two water treatment works (WTW);

2. Increased annual rehabilitation rate for pipes;

3. Addition of rainwater harvesting (RWH) and grey water recycling (GWR) schemes; 
Based on the above individual intervention options, the following seven UWS intervention strategies (alternatives) against the 30 year planning horizon (2011-2040) are proposed:

A0 Business as usual (BAU);

A1 Addition of a new water resource along with two WTWs starting from 2020;

A2 1\% additional annual pipe rehabilitation starting from 2015;

A3 Addition of RWH and GWR systems at a local level by $25 \%$ of households starting from 2015 ;

A4 Addition of RWH and GWR systems at a local level by $25 \%$ and $50 \%$ of households, respectively, starting from 2015 ;

A5 Addition of RWH and GWR systems at a local level by 50\% of households starting from 2015;

A6 Addition of RWH and GWR systems at a local level by $25 \%$ of households and $0.5 \%$ additional rehabilitation annually starting from 2015 ;

The first strategy assumes business as usual (BAU), i.e. effectively 'do nothing' in the UWS over the planning horizon 2011-2040. In fact, the BAU assumes there is no intervention options are added to the UWS over the planning horizon when the specific rate of population growth (high or low) is envisaged. Therefore, the performance of the other six intervention strategies (A1-6) comprised of at least one intervention option are compared to each other plus the first strategy. Note that the intervention strategies numbered A2 to A6 start from 2015 while strategy A1 starts from 2020. Applying each of these intervention strategies is expected to have some specific impacts on the performance metrics of the UWS. These performance metrics specified for this analysis are described in the following.

\section{Stakeholder Preferences}

Comparison of the intervention strategies with respect to the above performance metrics can be conducted based on either equal metric weights or some specific weighting schemes based on priorities of different groups/parties. For the sake of this analysis, three weighting schemes, including equal weights, Water Company and Consumer perspectives, are considered for ranking the intervention strategies (Table 2).

Table 2 Metric weighting schemes according to differening stakeholder preferences

\begin{tabular}{lcccccc}
\hline & \multicolumn{5}{c}{ Performance Metric } \\
\cline { 2 - 7 } & $\begin{array}{c}\text { Mo. Reliability } \\
\text { of Supply }\end{array}$ & $\begin{array}{c}\text { M1. Total } \\
\text { Costs }\end{array}$ & $\begin{array}{c}\text { M2. GHG } \\
\text { emissions }\end{array}$ & M3. Leakage & $\begin{array}{c}\text { M4. CSO } \\
\text { volume }\end{array}$ & $\begin{array}{c}\text { M5. Social } \\
\text { acceptance }\end{array}$ \\
\cline { 2 - 7 } W0. Equal weights & 1 & 1 & 1 & 1 & 1 & 1 \\
W1. Public & 4 & 1 & 3 & 4 & 3 & 1 \\
W2. Water company & 5 & 3 & 2 & 4 & 4 \\
\hline
\end{tabular}

\section{Population of Decision Matrix}

The time-series of the quantitative metrics (M0-M4) over the planning horizon are calculated by the DSS by running the WaterMet ${ }^{2}$ model with respect to each scenario and intervention strategy. The single value for each of these metrics is calculated and populates the Decision Matrix as presented in Table 3 and

Table 4 for each of the two scenarios. 
Table 3 Decision Matrix for Scenario S0 (low population growth)

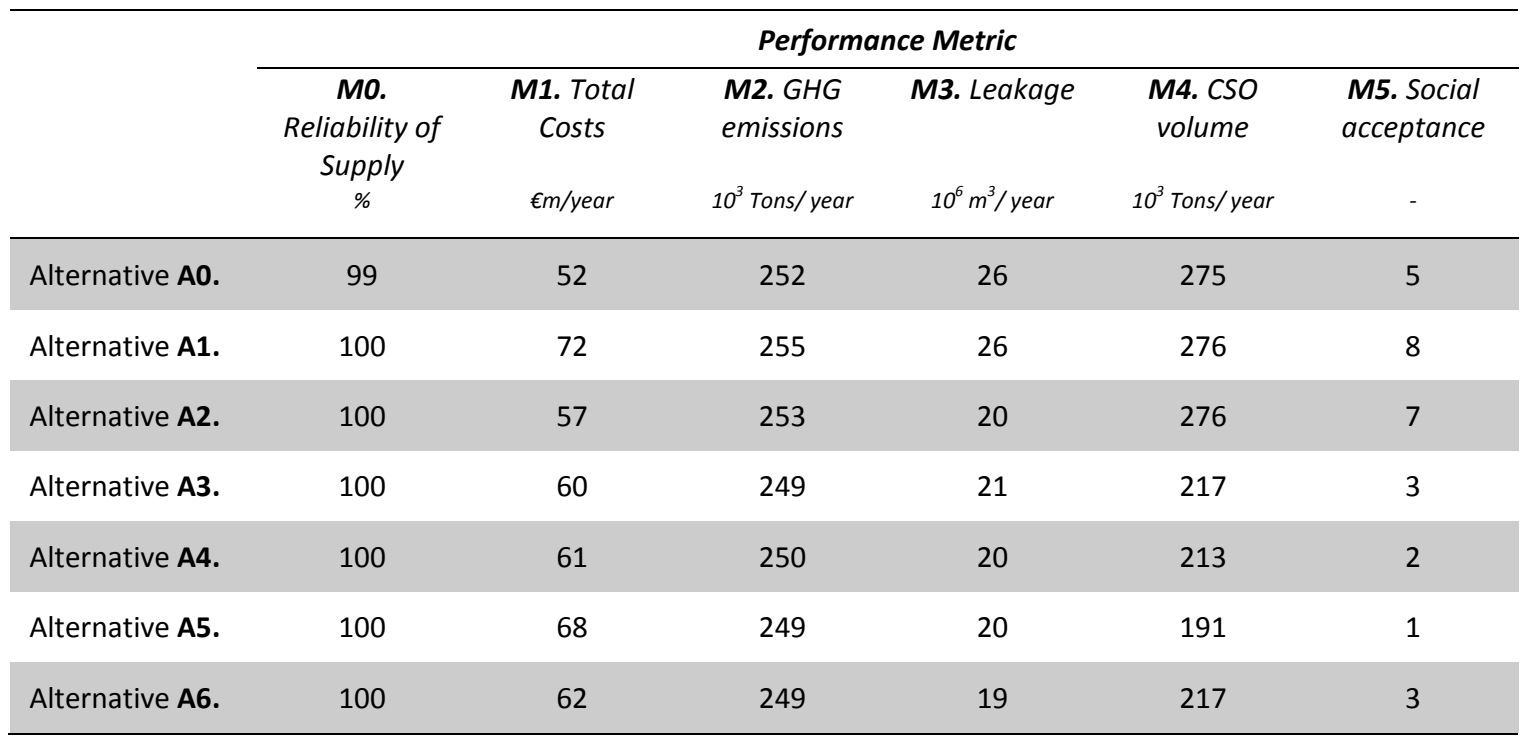

Table 4 Decision Matrix for Scenario S1 (high population growth)

\begin{tabular}{|c|c|c|c|c|c|c|}
\hline & \multicolumn{6}{|c|}{ Performance Metric } \\
\hline & $\begin{array}{c}\text { MO. } \\
\text { Reliability of } \\
\text { Supply }\end{array}$ & $\begin{array}{l}\text { M1. Total } \\
\text { Costs }\end{array}$ & $\begin{array}{l}\text { M2. GHG } \\
\text { emissions }\end{array}$ & M3. Leakage & $\begin{array}{l}\text { M4. CSO } \\
\text { volume }\end{array}$ & $\begin{array}{l}\text { M5. Social } \\
\text { acceptance }\end{array}$ \\
\hline & $\%$ & Em/year & $10^{3}$ Tons/year & $10^{6} \mathrm{~m}^{3} /$ year & $10^{3}$ Tons/year & - \\
\hline Alternative $\mathbf{A 0}$. & 95 & 53 & 273 & 29 & 289 & 5 \\
\hline Alternative $\mathbf{A} \mathbf{1}$. & 100 & 74 & 285 & 30 & 301 & 8 \\
\hline Alternative $\mathbf{A 2}$. & 97 & 58 & 277 & 23 & 293 & 7 \\
\hline Alternative $\mathbf{A} \mathbf{3}$. & 98 & 61 & 276 & 24 & 230 & 3 \\
\hline Alternative A4. & 99 & 62 & 278 & 23 & 226 & 2 \\
\hline Alternative $\mathbf{A} 5$. & 99 & 69 & 278 & 23 & 203 & 1 \\
\hline Alternative A6. & 99 & 63 & 277 & 22 & 231 & 3 \\
\hline
\end{tabular}

\section{Ranking of Alternatives}

Given the three weighting schemes and two scenarios, a total of six groups of ranking for the intervention strategies are obtained. Naturally, there are several ways that these rankings can be merged together to achieve a final ranking for each intervention strategy. In this instance, the sum of the ranks of each strategy is used for determining final ranking, as shown in the last column for each scenario in Table 5. 
Table 5 Summary of per-weighting rankings (Compromise Programming) of alternatives and overall ranking for each scenario

\begin{tabular}{|c|c|c|c|c|c|c|c|c|c|c|}
\hline & \multicolumn{5}{|c|}{ Scenario SO (Low Population Growth) } & \multicolumn{5}{|c|}{ Scenario S1 (High Population Growth) } \\
\hline & \multicolumn{3}{|c|}{ Weighting } & \multirow{2}{*}{$\begin{array}{l}\text { Sum } \\
\text { of } \\
\text { ranks }\end{array}$} & \multirow{2}{*}{$\begin{array}{l}\text { Final } \\
\text { ranking }\end{array}$} & \multicolumn{3}{|c|}{ Weighting } & \multirow{2}{*}{$\begin{array}{c}\text { Sum of } \\
\text { ranks }\end{array}$} & \multirow{2}{*}{$\begin{array}{l}\text { Final } \\
\text { ranking }\end{array}$} \\
\hline & $\begin{array}{c}\text { Wo. } \\
\text { Equal } \\
\text { weight }\end{array}$ & $\begin{array}{l}\text { W1. } \\
\text { Public }\end{array}$ & $\begin{array}{l}\text { W2. Water } \\
\text { Company }\end{array}$ & & & $\begin{array}{c}\text { wo. } \\
\text { Equal } \\
\text { weight }\end{array}$ & $\begin{array}{l}\text { W1. } \\
\text { Public }\end{array}$ & $\begin{array}{c}\text { W2. } \\
\text { Water } \\
\text { Company }\end{array}$ & & \\
\hline Alternative $\mathbf{A} \mathbf{0}$. & 5 & 3 & 3 & 11 & 3 & 4 & 3 & 2 & 9 & 2 \\
\hline Alternative $\mathbf{A} \mathbf{1}$. & 7 & 1 & 4 & 12 & 4 & 7 & 2 & 5 & 14 & 5 \\
\hline Alternative $\mathbf{A} \mathbf{2}$. & 1 & 2 & 1 & 4 & 1 & 1 & 1 & 1 & 3 & 1 \\
\hline Alternative $\mathbf{A} \mathbf{3}$. & 3 & 5 & 5 & 13 & 5 & 3 & 4 & 4 & 11 & 4 \\
\hline Alternative A4. & 4 & 6 & 6 & 16 & 6 & 5 & 6 & 6 & 17 & 6 \\
\hline Alternative $\mathbf{A 5}$. & 6 & 7 & 7 & 20 & 7 & 6 & 7 & 7 & 20 & 7 \\
\hline Alternative A6. & 2 & 4 & 2 & 8 & 2 & 2 & 5 & 3 & 10 & 3 \\
\hline
\end{tabular}

As can be seen, Alternative A2, which has been consistently ranked highly, is selected in the top Strategy for both scenarios. Alternative A5 has the lowest final rank because it has been identified as the worst strategy for several scenario/weighting combinations. Therefore, while it is sensible to recommend Alternatives A2, then A0/A6 as the best strategies to adopt in this simple example, Alternatives A4 and A5 are clearly not to be recommended. However, further analysis will be required to fully cover and test different criteria for these strategies.

\section{CONCLUSIONS}

A new DSS was developed to facilitate decision-making for the long-term city metabolism planning problem. This represents a novel methodology for comparison and selection of alternative intervention strategies, within the framework of long-term transition paths, accommodating multiple decision criteria and able to deal with uncertain future scenarios and differing stakeholder perspectives.

Both DSS methodology and software tool were described first in detail. Then, the effectiveness of the DSS was demonstrated on the northern European city case study. The case study involved the assessment of seven alternative intervention strategies in an UWS over a 30 year planning horizon. The DSS employs the WaterMet ${ }^{2}$ model which was used to calculate the six quantitative type metrics for the two scenarios of different future population growth. A further, qualitative type metric quantified by the experts outside the DSS was also included in the decision matrix to represent social acceptability of each intervention strategy. The DSS was then used to rank the intervention strategies using the Compromise Programming MCDA method for several different weighting schemes 
representing different stakeholder preferences. The most robust intervention strategy was then identified as the one that was ranked highly in all scenarios and for different stakeholder preferences. The results obtained on a case study demonstrate that the DSS developed and presented here can be used to effectively and efficiently assist the planners in making better, more objective and strategic level decisions with respect to meeting the long-term goals and performance targets in their Urban Water System.

\section{ACKNOWLEDGEMENTS}

This work was carried out as part of the 'TRansition to Urban water Services of Tomorrow' (TRUST) project funded in the EU $7^{\text {th }}$ Framework Programme under Grant Agreement No. 265122. The authors also wish to thank NTNU, Oslo VAV, LNEC and Addition (TRUST project partners) for their collaboration.

\section{REFERENCES}

Alegre, H., Cabrera jr., E., Hein, A. and Brattebø, H. (2012), Framework for Sustainability Assessment of UWCS and development of a self-assessment tool. Deliverable D31.1. TRUST Project.

Behzadian, K., and Kapelan, Z., (2013), Oslo Case Study Report, University of Exeter, Exeter, UK, TRUST Project.

Behzadian, K., Kapelan, Z., Rozos, E. and Makropoulos, C. (2013a), WaterMet ${ }^{2}$ conceptual model report. Deliverable D33.1. TRUST Project.

Behzadian, K., Kapelan, Z., Venkatesh, G., Brattebø, H., Sægrov, S., Rozos, E., Makropoulos, C., Ugarelli, R., Milina, J. and Hem, L. (2013b), Urban water system metabolism assessment using WaterMet ${ }^{2}$ model, 12th International Conference on Computing and Control, Italy, vol. 15.

Hardy, M.J., Kuczera, G. and Coombes, P.J. (2005), Integrated urban water cycle management: the urban cycle model. Water Science \& Technology, 52(9).

Mackay, R. and Last, E. (2010), SWITCH city water balance: a scoping model for integrated urban water management. Reviews in Environmental Science and Biotechnology, 9(4), pp291-296.

Makropoulos, C.K., Natsis, K., Liu, S., Mittas, K. and Butler, D. (2008), Decision support for sustainable option selection in integrated urban water management. Environmental Modelling \& Software, 23(12), pp1448-1460.

Mitchell, V.G., Mein, R.G. and McMahon, T.A. (2001), Modelling the urban water cycle. Environmental Modelling \& Software, 16(7) pp615-629.

Mitchell, V.G. and Diaper, C. (2010), UVQ User Manual: (urban water balance and contaminant balance analysis tool), Version 1.2, CMIT Report No. 2005-282. CSIRO.

Morley, M.S., Kapelan, Z. and Savić, D.A. (2012), Integrated Decision Support Framework. Deliverable D54.1. TRUST Project.

Saaty, T.L. (1980). The Analytic Hierarchy Process: Planning, Priority Setting, Resource Allocation, ISBN 0-07-054371-2, McGrawHill, New York, U.S.A. 287pp.

Venkatesh, G., Sægrov, S. and Brattebø, H. (2014), Dynamic metabolism modelling of urban water services - Demonstrating effectiveness as a decision-support tool for Oslo, Norway. Water Res.; 61, pp19-33.

Willuweit, L. and O'Sullivan, J.J. (2013). A decision support tool for sustainable planning of urban water systems: Presenting the Dynamic Urban Water Simulation Model. Water Research, 47(20), pp7206-7220.

Zeleny, M., (1973). Compromise Programming. In: Multiple Criteria Decision Making, Cochrane and M. Zeleny (Editors), University of South Carolina Press: Columbia, South Carolina. 